\title{
Bateria Multidimensional de Inteligência Infantil: desenvolvimento de instrumento
}

\author{
Patricia Waltr. Schelini \\ Solange Wechsler
}

\begin{abstract}
Resumo
O estudo objetivou elaborar um conjunto de testes, denominado "Bateria Multidimensional de Inteligência Infantil" ou BMI, para avaliar capacidades do Modelo Cattell-Horn-Carroll. Entre as capacidades avaliadas estão as de inteligência cristalizada, inteligência fluida, velocidade de processamento cognitivo, memória a curto prazo, armazenamento e recuperação associativa a longo prazo e conhecimento quantitativo. A BMI foi composta por nove testes, apresentados a duas amostras de participantes. A primeira foi formada por 240 crianças, com idade entre sete e 12 anos. Constituída para que novos itens fossem testados, a segunda amostra foi formada por outras 206 crianças. Os resultados demonstraram a influência altamente significativa da idade sobre o desempenho dos testes. Os testes Informação Geral, Indução, Desempenho em Matemática, Vocabulário Geral e Vocabulário Ilustrado apresentaram elevados coeficientes de precisão. A análise dos índices de dificuldade e do poder discriminativo permitiu a seleção do conjunto mais adequado de questões para compor a configuração final da Bateria.

Palavras-Chave: Inteligência; Testes; Modelo Cattell-Horn-Carroll, Avaliação psicológica.
\end{abstract}

\section{Multidimensional Battery for Children's Intelligence: Development of an instrument}

\begin{abstract}
The goal of this study was elaborate a set of tests, "Multidimensional Battery for Children's Intelligence" or "BMI", according to the abilities included in the Cattell-Horn-Carroll's Model. Among these abilities are the ability of crystallized intelligence, fluid intelligence, processing speed, short-term memory, long-term storage and retrieval and quantitative knowledge. The BMI was composed by nine tests, presented to two samples of participants. The first sample comprised 240 children whose age ranged from seven to 12 years old. Constituted for the testing of new items, the second sample comprised another 206 children. The results demonstrated that the age had a high influence on the tests performance. Were found high reliability coefficients for the tests General Information, Induction, Mathematics Achievment, General Vocabulary and Picture Vocabulary. The analyses of their difficulty levels and their discriminitative power led to the choice of the best set of questions to integrate the final configuration of the Battery.
\end{abstract}

Keywords: Intelligence; Tests; Cattell-Horn-Carroll's model; Psychological assessment.

\section{Introdução}

No início do século XX, o psicólogo britânico Charles Spearman apresentou a Teoria dos Dois Fatores da Inteligência (Thorndike, 1997). Esta teoria, também conhecida como Bi-Fatorial, postulava que o desempenho em qualquer medida de inteligência estaria relacionado ao nível de inteligência geral do indivíduo e a habilidades específicas exigidas em cada teste (Aiken, 2000; Cronbach, 1996).

Em 1909, Thorndike, Lay e Dean, analisando a existência do fator $g$ em um conjunto de medidas semelhante ao utilizado por Spearman, concluíram que não havia indícios suficientes para sustentar o sistema Bi-Fatorial. Na década de trinta, Louis Thurstone propôs a existência de um pequeno número de fatores independentes ou capacidades mentais primárias
(Gardner, Kornhaber \& Wake, 1998).

Na década de 40 e, principalmente, nos anos 50 e 60 alguns autores elaboraram concepções capazes de conciliar as abordagens anteriormente citadas (Almeida, 1988). Nesta perspectiva surge a Teoria das Inteligências Fluida e Cristalizada.

Raymond Cattell, em 1942, analisando as correlações entre as capacidades primárias de Thurstone e o fator $g$ da Teoria Bi-Fatorial, constatou a existência de dois fatores gerais. Alguns anos depois, John Horn confirmou os estudos de Cattell e designou os fatores gerais como "inteligência fluida e cristalizada" (Cattell, 1987, 1998).

A inteligência fluida $(G f)$ está associada a componentes não-verbais, pouco dependendo de conhecimentos previamente adquiridos. As operações mentais que as pessoas utilizam diante de uma tarefa que não

${ }^{1}$ Endereço para correspondência:

Universidade Federal de São Carlos - UFSCar/ Departamento de Psicologia

Rodovia Washington Luís, km 235 - Caixa Postal 676 - Monjolinho - 13565-905 - São Carlos-SP 
pode ser executada automaticamente representam $G f$ (Horn, 1991; Kline, 2000). Por outro lado, a inteligência cristalizada $(G c)$ representa tipos de capacidades exigidas na solução da maioria dos complexos problemas cotidianos, sendo desenvolvida com base em experiências culturais (Campito, 1994; Horn, 1991). Em 1965, Horn iniciou a expansão do modelo proposto por Cattell, acrescentando oito fatores gerais às duas capacidades básicas $(G f$ e $G c)$.

John Carroll (1993) propôs uma estrutura hierárquica para a compreensão das capacidades cognitivas: a Teoria das Três Camadas, que diferenciou as capacidades por meio de três diferentes camadas: a camada I, de capacidades específicas; a II, formada por capacidades amplas ou gerais; e a III, relativa a uma única capacidade geral.

Apesar das diferenças entre os modelos de Carroll e Horn-Cattell, McGrew e Flanagan (1998) sintetizaram estas duas perspectivas, originando o Modelo Cattell-Horn-Carroll, fundamento teórico da Bateria Multidimensional de Inteligência Infantil (BMI), cuja elaboração foi o principal objetivo do presente estudo.

O modelo das Capacidades Cognitivas de CattellHorn-Carroll (CHC) estabelece a existência de dez capacidades gerais, dispostas na camada II, entre elas: inteligência fluida $(G f)$, conhecimento quantitativo $(G q)$, inteligência cristalizada $(G c)$, memória a curto prazo
$(G s m)$, processamento visual $(G v)$, processamento auditivo $(G a)$, armazenamento e recuperação associativa a longo prazo (Glr), velocidade de processamento cognitivo $(G s)$, tempo/velocidade de decisão/reação $(G t) \mathrm{e}$ leitura-escrita $(G m w)$. Cada fator geral da camada II é formado por fatores específicos, estabelecidos na camada I. Desse modo, o fator geral de inteligência fluida $(G f)$, por exemplo, é composto pelos fatores específicos de raciocínio seqüencial geral $(R G)$, indução $(I)$, raciocínio quantitativo $(R Q)$, raciocínio piagetiano $(R P)$ e velocidade de raciocínio (RE).

Algumas capacidades específicas relacionadas às capacidades gerais de inteligência cristalizada $(G c)$, inteligência fluida $(G f)$, conhecimento quantitativo $(G q)$, memória a curto prazo (Gsm), armazenamento e recuperação associativa a longo prazo $(G l r)$ e velocidade de processamento cognitivo (Gs) foram escolhidas, dentre as demais descritas no modelo de Cattell-HornCarroll, para ser avaliadas por testes que irão compor a Bateria Multidimensional de Inteligência Infantil (BMI). Isto porque estas capacidades gerais são indicadas como as mais relacionadas às exigências escolares, especialmente no que diz respeito à leitura e à matemática (Flanagan, Andrews \& Genshaft, 1997; Flanagan \& Ortiz, 2001; Friedman, 1995; Rasanen \& Ahonen, 1995). A tabela seguinte apresenta, de forma resumida, as definições das capacidades específicas do modelo Cattell-Horn-Carroll enfatizadas neste estudo.

Tabela 1 - Descrição das capacidades específicas avaliadas pela BMI

\begin{tabular}{|c|c|}
\hline Capacidades específicas & Descrição \\
\hline Informação geral $(K O)$ & Quantidade de conhecimentos gerais adquiridos. Avaliada pelo teste Informação Geral. \\
\hline ento léxico $(V L)$ & $\begin{array}{l}\text { Extensão do vocabulário ou conhecimento do significado das palavras. Avaliado } \\
\text { pelos testes Vocabulário Geral e Vocabulário Ilustrado. }\end{array}$ \\
\hline $\begin{array}{l}\text { Desenvolvimento da } \\
\text { linguagem }(L D)\end{array}$ & $\begin{array}{l}\text { Desenvolvimento geral da língua nativa, referindo-se, ainda, ao entendimento de } \\
\text { palavras, sentenças e parágrafos na verbalização. Avaliada pelos testes Vocabulário } \\
\text { Geral e Vocabulário Ilustrado. }\end{array}$ \\
\hline Indução $(I)$ & $\begin{array}{l}\text { Capacidade de descobrir as características (regra, conceito, processo, relação de } \\
\text { causa) que governam um problema ou um conjunto de materiais. Avaliada pelo teste } \\
\text { Indução. }\end{array}$ \\
\hline Desempenho matemático $(A 3)$ & $\begin{array}{l}\text { Quantidade de desempenhos matemáticos. Avaliado pelo teste Desempenho em } \\
\text { Matemática. }\end{array}$ \\
\hline Extensão da memória (MS) & $\begin{array}{l}\text { Quantidade de elementos (verbais, numéricos ou figurais) que a pessoa consegue } \\
\text { recordar, imediatamente após serem apresentados. Avaliada pelo teste Memória } \\
\text { para Nomes. }\end{array}$ \\
\hline Memória associativa $(M A)$ & $\begin{array}{l}\text { Capacidade de lembrar de um elemento ou parte de pares de itens quando a outra } \\
\text { parte ou elemento estiver presente. Avaliada pelo teste Memória Associativa. }\end{array}$ \\
\hline $\begin{array}{l}\text { Velocidade de resposta } \\
\text { ao teste }(R 9)\end{array}$ & $\begin{array}{l}\text { Capacidade de realizar rapidamente testes relativamente fáceis ou que exijam } \\
\text { decisões muito simples. Avaliada pelos testes Velocidade de Percepção e Rapidez } \\
\text { de Resposta. }\end{array}$ \\
\hline Velocidade perceptual $(P)$ & $\begin{array}{l}\text { Capacidade de rapidamente procurar e comparar símbolos visuais apresentados lado a } \\
\text { lado ou separados em um campo visual. Avaliada pelo teste Velocidade de Percepção. }\end{array}$ \\
\hline
\end{tabular}




\section{Objetivos}

O presente trabalho é decorrente do estudo realizado por Schelini (2002) e teve como principal objetivo a construção de um instrumento destinado à avaliação da inteligência de crianças de 7 a 12 anos Deste modo, os objetivos específicos deste projeto foram: elaborar um instrumento que avaliasse a inteligência por meio das capacidades gerais de inteligência cristalizada, inteligência fluida, velocidade de processamento cognitivo, memória a curto prazo, armazenamento e recuperação associativa a longo prazo e conhecimento quantitativo; verificar a validade de construto do instrumento pela diferenciação por idade e verificar a precisão do instrumento.

\section{Método}

\section{Participantes}

A primeira amostra de participantes foi constituída por 240 crianças de sete a 12 anos de idade, distribuídas em seis faixas etárias com amplitude de um ano. Cada faixa etária foi representada por 40 crianças, 20 do sexo feminino e 20 do sexo masculino. Em cada faixa etária do sexo feminino, 14 crianças eram estudantes de escolas públicas e seis de escolas particulares, o mesmo ocorrendo em relação ao sexo masculino. Um total de 12 escolas, localizadas em sete cidades do interior do estado de São Paulo, colaboraram para a formação da primeira amostra.

A segunda amostra foi constituída para que novos itens, criados após a análise estatística dos dados da primeira amostra, pudessem ser testados. Esta amostra foi formada por outras 206 crianças de sete a 12 anos de idade, distribuídas em seis faixas etárias com amplitude de um ano. Cada faixa etária foi representada por uma média de 34 crianças, sendo 17 do sexo feminino e 17 do sexo masculino. Em cada faixa etária do sexo feminino, aproximadamente 14 crianças eram estudantes de escolas públicas e três de particulares, o mesmo ocorrendo em relação ao sexo masculino. Nove escolas, localizadas em cinco cidades do interior do estado de São Paulo, colaboraram para a formação desta amostra.

\section{Material}

O material (Bateria Multidimensional de Inteligência Infantil) foi composto por nove testes destinados a avaliar capacidades relativas ao modelo Cattell-HornCarroll (CHC). Ao elaborar os testes formados por itens com diferentes níveis de dificuldade, houve a preocupação em criar aproximadamente o dobro da quantidade total de itens que iria compor as versões finais dos testes, afinal, há uma tendência de muitos itens serem considerados ineficazes por possuir um reduzido poder discriminativo.

A seguir, os instrumentos que compõem a Bateria Multidimensional de Inteligência Infantil (BMI) são descritos na ordem em que foram apresentados às amostras 1 e 2.

\section{Teste 1: Informação Geral}

Este teste foi elaborado com o objetivo de avaliar a quantidade de conhecimentos gerais apreendidos do meio, sendo formado inicialmente por 42 questões apresentadas oralmente sobre conhecimentos relativos a objetos, lugares, eventos e pessoas. Após a análise estatística das respostas dos participantes da primeira amostra, sete itens foram adicionados para que uma melhor distribuição quanto à dificuldade fosse alcançada. Desta forma, 49 itens foram testados na segunda amostra.

As instruções gerais são: "Vou fazer algumas perguntas e gostaria que você respondesse da melhor maneira possível. Tente dar a sua melhor resposta". Depois disso, dois exemplos são apresentados. Alguns exemplos de itens que compõem o teste Informação Geral são citados a seguir.

1. O examinador aponta para uma de suas orelhas e pergunta: Como se chama isto?

28. Você poderia dizer o nome de dois planetas? humano?

41. Qual o nome do maior osso do corpo

42. Quem foi Carlos Gomes?

\section{Teste 2: Memória Associativa}

O Memória Associativa objetiva avaliar a habilidade em memorizar associações entre estímulos visuais. Para isso são apresentados doze extraterrestres, ao lado de seus respectivos planetas. Em seguida, é apresentado um extraterrestre e o participante deve localizar, no meio de vários planetas, qual o planeta deste extraterrestre. Assim, o teste é formado por 12 itens, não tendo sido adicionado nenhum depois da análise das respostas da primeira amostra. 


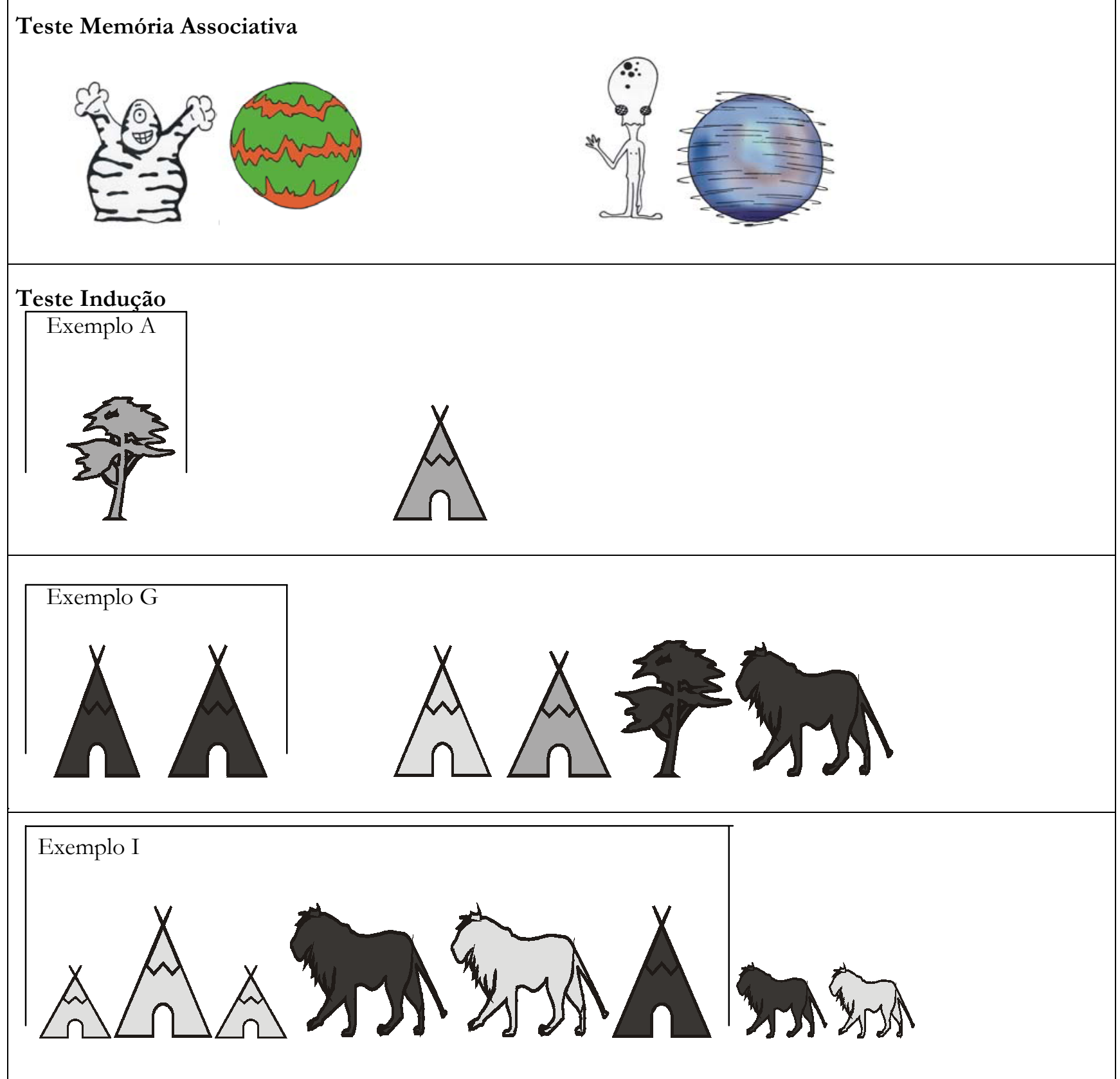

Figura 1 - Teste Memória Associativa e Indução: exemplos

\section{Teste 3: Inducão}

Este teste, parcialmente elaborado com a colaboração do dr. Ricardo Primi, visa avaliar o raciocínio indutivo mediante a capacidade de identificar regras para a formação de conceitos. Com tal objetivo são apresentadas figuras de diferentes formas, cores e tamanhos e o participante deve dizer qual o critério (tamanho, forma, cor) responsável pelo fato de o grupo de figuras de dentro do quadro ser diferente do grupo de fora.

No início, o examinador apresenta a folha onde estão contidos todos os tipos de figuras que aparecem no teste, em suas diversas formas, cores e tamanhos.
No exemplo A (ver Tabela 2) o examinador explica que a figura de dentro do quadro é diferente da que está fora por sua forma. Outros cinco exemplos são dados e, em seguida, é iniciada a apresentação dos itens 1 ao 18.

A partir dos exemplos $\mathrm{G}$ e $\mathrm{H}$, dois critérios atuam, ao mesmo tempo, de modo a diferenciar as figuras de dentro do quadro das de fora. Assim, no exemplo $G$ as figuras de dentro são diferentes das de fora por causa da forma e da cor. Itens com estas características são apresentados até que nos exemplos I e J é explicado que as figuras de dentro do quadro são diferentes das de fora por causa da cor ou do tamanho, da cor ou da 
forma, da forma ou do tamanho. Deste modo, no exemplo I deve ficar claro que as figuras de dentro são diferentes das de fora devido ao tamanho ou à forma.

O teste foi inicialmente formado por 26 itens. Após a análise das respostas da primeira amostra, 14 itens foram incluídos, entre eles os que envolvem o tipo de raciocínio exigido no exemplo $G$.

\section{Teste 4: Memória para Nomes}

O Memória para Nomes busca avaliar a habilidade de prestar atenção e recordar de modo imediato a ordem de elementos apresentados em uma única vez. É composto por 15 seqüências de nomes (substantivos de duas sílabas) que o participante deve repetir oralmente e de maneira idêntica a que foram apresentadas. Vale ressaltar que a escolha dos nomes baseou-se no estudo de Pinheiro (1996), de modo que foram selecionadas palavras com alta freqüência de ocorrência entre estudantes do Ensino Fundamental.

Exemplos de itens na ordem da apresentação:

1. bicho-pedra

3. casa-roda

13. maçã-café-vela-rosto-fogão-prova

15. anjo-sapo-festa-prédio-garfo-banco

\section{Teste 5: Desempenho em Matemática}

Com o objetivo de avaliar a habilidade de realizar operações matemáticas, um conjunto de problemas aritméticos deve ser resolvido e respondido oralmente, com o auxílio de lápis e papel. Um total de 42 itens foram testados na primeira amostra e, após a análise das respostas, seis itens foram adicionados.

Exemplos de itens na ordem da apresentação:

1. Você comeu uma fruta de manhã e à noite comeu outra fruta. Quantas frutas comeu no total? bolas sobraram?

3. Lúcia tinha 4 bolas. Ela perdeu uma. Quantas

41. Quando Cláudia sobe em um banquinho de 0,3 metros ela fica com 1,93 metros de altura. Qual é a altura de Cláudia?

48. Paulo comprou uma máquina fotográfica usada por $\mathrm{R} \$ 36,00$. Ele pagou $2 / 3$ do preço de uma máquina nova. Quanto custa uma máquina nova?

\section{Teste 6: Vocabulário Geral}

Avaliar a extensão do vocabulário, o conhecimento do significado das palavras e a qualidade do desenvolvimento da linguagem são objetivos do Vocabulário Geral. Assim, um conjunto de palavras é apresentado oralmente e seus significados devem ser explicados. Um total de 47 itens foi aplicado na primeira amostra, cinco novos itens foram elaborados, de modo que 52 questões foram testadas na segunda amostra.
Exemplos de itens na ordem da apresentação:

5. O que é um sapo?

10. O que é um colega?

42. O que significa pacto?

47. O que significa amparo?

\section{Teste 7: Velocidade de Percepscão}

A avaliação da habilidade em rapidamente localizar e assinalar determinado conjunto de símbolos numéricos é um dos objetivos deste teste, em que o examinando assinala quatro conjuntos de números que, idênticos ao modelo, estão dispostos em uma fileira com outros conjuntos numéricos. A atividade deve ser feita da forma mais rápida possível pela existência de um limite de tempo de 120 segundos (dois minutos).

\section{Teste 8: Vocabulário Ilustrado}

Este teste objetiva analisar a extensão do vocabulário e a qualidade do desenvolvimento da linguagem pela habilidade em reconhecer e nomear ilustrações. Um conjunto de ilustrações a serem identificadas ou nomeadas caracterizam o Vocabulário Ilustrado. Na primeira amostra foram aplicados 55 itens; outros 38 foram elaborados, originando 93 a serem testados na segunda amostra.

Em seguida são citados alguns exemplos de itens na ordem da apresentação.

53. O que é isto? (esfinge)

54. O que é isto? (balança) (astronauta)

65. Quem é este homem? Qual a profissão dele?

77. Como se chama esta parte do olho? (o examinador deve apontar a pupila)

\section{Teste 9: Rapider de Resposta}

O teste Rapidez de Resposta foi elaborado para avaliar a habilidade em associar rapidamente uma cor a um estímulo. Com este objetivo, duas cores (azul e vermelho) estão relacionadas a estímulos simples (lápis, carro, coração e pessoa). O participante deve associar as cores aos estímulos da maneira mais rápida possível em um período de tempo de 60 segundos (um minuto).

As seguintes instruções são apresentadas: "Olhe para estes desenhos (o examinador aponta a parte superior da folha onde está o modelo). Você pode ver um lápis azul, um carro também azul, um coração vermelho e uma pessoa em vermelho. Agora olhe aqui embaixo. Você pode ver os mesmos desenhos, só que eles não estão coloridos, estão todos em preto". Uma caneta azul e uma vermelha são dadas, de modo que o coração e a pessoa sejam assinalados em vermelho e o lápis e o carro em azul.

\section{Procedimento}

Anteriormente ao início da coleta de dados foi 
realizada uma pré-testagem, com o objetivo de verificar se as instruções e itens dos instrumentos da bateria eram compreensíveis às várias faixas etárias. Finalizado o pré-teste, foram definidas as escolas públicas e particulares que participaram da primeira amostra. O critério adotado para a definição das escolas foi, principalmente, a localização das instituições, uma vez que houve a tentativa de selecionar escolas situadas nas mais variadas regiões das cidades. Definidas as escolas, os objetivos e as características da pesquisa foram esclarecidos aos diretores e coordenadores, sendo a eles solicitada uma autorização para a entrada na instituição. Os nomes dos alunos de sete a 12 anos foram listados, sendo sorteados os 240 participantes da primeira amostra. Um pedido de autorização aos responsáveis pelos alunos foi enviado e, depois disso, foi iniciada a coleta de dados.

Os instrumentos foram apresentados na seguinte ordem: Informação Geral, Memória Associativa, Indução, Memória para Nomes, Desempenho em Matemática, Vocabulário Geral, Velocidade de Percepção, Vocabulário Ilustrado e Rapidez de Resposta. Vale dizer que todos os itens foram aplicados, não havendo interrupção de qualquer teste.

Terminada a aplicação da bateria na primeira amostra, foi realizada a avaliação das respostas e a análise estatística dos dados. Com base nesta primeira análise estatística foi constatada a importância de elaboração de novos itens para alguns testes (Informação Geral, Indução, Desempenho em Matemática, Vocabulário Geral e Vocabulário Ilustrado), de modo que houvesse uma adequada distribuição quanto à dificuldade.

Após a elaboração de novos itens e a autorização do estudo nas escolas, os nomes de outros alunos de sete a 12 anos foram listados, sendo sorteados os participantes da segunda amostra. Um pedido de autorização aos responsáveis pelos alunos foi enviado e, depois disso, foi iniciada a coleta de dados. Depois de aplicar a bateria na segunda amostra foi realizada a avaliação das respostas.

\section{Resultados}

Os resultados referentes às respostas dos participantes da segunda amostra são apresentados a seguir. Isto porque os participantes desta amostra responderam a todos os itens, inclusive àqueles elaborados depois $\mathrm{da}$ análise estatística das respostas da primeira amostra de participantes.

Tabela 2 - Médias e desvios padrão por idade nos testes da BMI

\begin{tabular}{|c|c|c|c|c|c|c|c|c|c|c|c|c|}
\hline \multirow{2}{*}{$\frac{\text { Idade }}{\text { Testes }}$} & \multicolumn{2}{|c|}{7 anos } & \multicolumn{2}{|c|}{8 anos } & \multicolumn{2}{|c|}{9 anos } & \multicolumn{2}{|c|}{10 anos } & \multicolumn{2}{|c|}{11 anos } & \multicolumn{2}{|c|}{12 anos } \\
\hline & $\mathrm{X}$ & $D P$ & $\overline{\mathrm{X}}$ & $D P$ & $\overline{\mathrm{X}}$ & $D P$ & $\mathrm{X}$ & $D P$ & $\mathrm{X}$ & $D P$ & $\mathrm{X}$ & $D P$ \\
\hline nformacão geral & 10,11 & 4,74 & 15,20 & 6,78 & 20,61 & 8,47 & 25,02 & 9,17 & 30,87 & 7,41 & 33,76 & 8,67 \\
\hline nem & 4,41 & 2,0 & 4,58 & 2,03 & 5,67 & 2,91 & 6,40 & 3,14 & 6,63 & 2,89 & 8,00 & 2,68 \\
\hline & 22,72 & 6,23 & 25,00 & 5,97 & 24,94 & 6,77 & 24,74 & 6,54 & 27,27 & 5,16 & 28,58 & 5,15 \\
\hline a para nomes & 7,91 & 1,66 & 8,26 & 1,97 & 8,47 & 1,65 & 9,11 & 1,56 & 9,18 & 1,42 & 9,88 & 1,82 \\
\hline $\begin{array}{l}\text { Desempenho em } \\
\text { matemática }\end{array}$ & 13,63 & 6,55 & 21,11 & 8,92 & 24,44 & 11,49 & 28,40 & 10,71 & 33,78 & 5,81 & 35,97 & 7,67 \\
\hline orer & 34,61 & 2,8 & 44,14 & 13,49 & 51,23 & 14,8 & 57,51 & 16, & 66,30 & 15,44 & 75,05 & 13,53 \\
\hline lade de percepção & 24,61 & 8,14 & 30,00 & 9,11 & 36,85 & 9,04 & 42,40 & 12,17 & 46,63 & 8,62 & 54,32 & 9,73 \\
\hline Vocabulário ilustrado & 112,76 & 20,95 & 120,04 & 21,86 & 130,77 & 22,20 & 139,97 & 17,57 & 150,36 & 16,62 & 160,98 & 12,19 \\
\hline Rapidez de resposta & 25,05 & 6,28 & 26,58 & 7,90 & 31,47 & 5,76 & 33,65 & 7,41 & 41,93 & 11,42 & 44,85 & 9,81 \\
\hline
\end{tabular}

A Tabela 2 sugere uma tendência de aumento das médias das pontuações dos testes proporcional às idades.

Tabela 3 - Análise da variância univariada por idade nos testes da BMI

\begin{tabular}{lcccc}
\hline Testes & Soma dos quadrados & DF & F & Significância \\
\hline Informação geral & 14309,26 & 5 & 48,56 & 0,000 \\
Memória associativa & 315,71 & 5 & 8,96 & 0,000 \\
Indução & 744,92 & 5 & 4,11 & 0,001 \\
Memória para nomes & 89,61 & 5 & 6,24 & 0,000 \\
Desempenho em matemática & 11975,65 & 5 & 31,05 & 0,000 \\
Vocabulário geral & 37531,87 & 5 & 35,49 & 0,000 \\
Velocidade de percepção & 20676,58 & 5 & 45,16 & 0,000 \\
Vocabulário ilustrado & 57562,23 & 5 & 32,12 & 0,000 \\
Rapidez de resposta & 11046,21 & 5 & 32,08 & 0,000 \\
\hline
\end{tabular}


Observa-se, por meio da Tabela 3, que todos os níveis de significância são menores ou iguais a $0,1 \%$. Isto indica que em todos os testes pelo menos um par de médias por idade é diferente, havendo um efeito significativo desta variável (idade).
Os testes da Bateria Multidimensional de Inteligência Infantil foram avaliados por meio das porcentagens de acertos, das correlações item-total e dos coeficientes de consistência interna. Tais resultados são apresentados em seguida.

Tabela 4 - Testes Informação Geral, Memória Associativa, Indução e Memória para Nomes: porcentagem de acertos, correlações item-total e coeficientes de consistência interna

\begin{tabular}{|c|c|c|c|c|c|c|c|c|c|c|c|c|}
\hline & Item & $\%$ & $\begin{array}{l}\text { Correl. } \\
\text { item-total }\end{array}$ & $\begin{array}{l}\text { Alpha se item } \\
\text { excluído }\end{array}$ & Item & $\%$ & $\begin{array}{c}\text { Correl. } \\
\text { item-total }\end{array}$ & $\begin{array}{l}\text { Alpha se item } \\
\text { excluído }\end{array}$ & Item & $\%$ & $\begin{array}{c}\text { Correl. } \\
\text { item-total }\end{array}$ & $\begin{array}{l}\text { Alpha se item } \\
\text { excluído }\end{array}$ \\
\hline \multirow{17}{*}{ 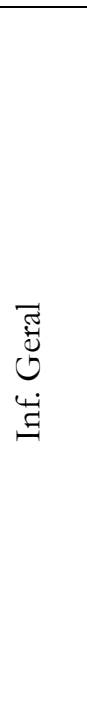 } & 1 & 97,57 & 0,10 & 0,95 & 18 & 49,03 & 0,67 & 0,94 & 34 & 18,45 & 0,59 & 0,94 \\
\hline & 2 & 97,57 & 0,14 & 0,95 & 19 & 45,63 & 0,67 & 0,94 & 35 & 16,99 & 0,51 & 0,94 \\
\hline & 3 & 98,54 & 0,13 & 0,95 & 20 & 32,04 & 0,56 & 0,94 & 36 & 12,14 & 0,56 & 0,94 \\
\hline & 4 & 92,72 & 0,21 & 0,95 & 21 & 38,83 & 0,48 & 0,95 & 37 & 26,70 & 0,64 & 0,94 \\
\hline & 5 & 84,95 & 0,45 & 0,95 & 22 & 46,12 & 0,56 & 0,94 & 38 & 20,87 & 0,58 & 0,94 \\
\hline & 6 & 72,82 & 0,43 & 0,95 & 23 & 84,47 & 0,35 & 0,95 & 39 & 14,08 & 0,48 & 0,95 \\
\hline & 7 & 74,27 & 0,45 & 0,95 & 24 & 26,21 & 0,40 & 0,95 & 40 & 16,99 & 0,52 & 0,94 \\
\hline & 8 & 42,23 & 0,44 & 0,95 & 25 & 14,56 & 0,60 & 0,94 & 41 & 7,28 & 0,38 & 0,95 \\
\hline & 9 & 51,94 & 0,65 & 0,94 & 26 & 14,08 & 0,36 & 0,95 & 42 & 5,34 & 0,36 & 0,95 \\
\hline & 10 & 83,50 & 0,43 & 0,95 & 27 & 9,71 & 0,37 & 0,95 & 43 & 60,68 & 0,67 & 0,94 \\
\hline & 11 & 64,56 & 0,59 & 0,94 & 28 & 66,99 & 0,71 & 0,94 & 44 & 17,48 & 0,43 & 0,95 \\
\hline & 12 & 46,12 & 0,54 & 0,94 & 29 & 24,76 & 0,61 & 0,94 & 45 & 47,09 & 0,68 & 0,94 \\
\hline & 13 & 64,56 & 0,64 & 0,94 & 30 & 34,47 & 0,59 & 0,94 & 46 & 77,67 & 0,58 & 0,94 \\
\hline & 14 & 60,19 & 0,65 & 0,94 & 31 & 50,49 & 0,55 & 0,94 & 47 & 48,54 & 0,54 & 0,94 \\
\hline & 15 & 73,30 & 0,48 & 0,95 & 32 & 24,27 & 0,63 & 0,94 & 48 & 29,13 & 0,58 & 0,94 \\
\hline & 16 & 46,60 & 0,57 & 0,94 & 33 & 35,92 & 0,65 & 0,94 & 49 & 42,72 & 0,43 & 0,95 \\
\hline & 17 & 33,98 & 0,41 & 0,95 & & & & & & & & \\
\hline \multirow{4}{*}{ 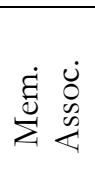 } & 1 & 84,95 & 0,34 & 0,74 & 5 & 50,97 & 0,48 & 0,72 & 9 & 30,10 & 0,35 & 0,73 \\
\hline & 2 & 68,45 & 0,46 & 0,72 & 6 & 39,81 & 0,38 & 0,73 & 10 & 25,73 & 0,25 & 0,75 \\
\hline & 3 & 70,39 & 0,42 & 0,73 & 7 & 50,49 & 0,42 & 0,73 & 11 & 58,25 & 0,39 & 0,73 \\
\hline & 4 & 55,34 & 0,42 & 0,73 & 8 & 26,70 & 0,34 & 0,74 & 12 & 32,52 & 0,32 & 0,74 \\
\hline \multirow{14}{*}{$\begin{array}{l}\text { 鹙 } \\
\stackrel{\Xi}{\Xi} \\
\end{array}$} & 1 & 100,00 & 0,00 & 0,83 & 15 & 63,59 & 0,34 & 0,82 & 28 & 63,59 & 0,16 & 0,83 \\
\hline & 2 & 96,12 & 0,18 & 0,83 & 16 & 56,80 & 0,26 & 0,83 & 29 & 69,42 & 0,42 & 0,82 \\
\hline & 3 & 98,06 & 0,28 & 0,83 & 17 & 61,17 & 0,36 & 0,82 & 30 & 42,23 & 0,25 & 0,83 \\
\hline & 4 & 97,57 & 0,31 & 0,83 & 18 & 66,02 & 0,39 & 0,82 & 31 & 58,25 & 0,35 & 0,82 \\
\hline & 5 & 95,63 & 0,22 & 0,83 & 19 & 48,54 & 0,35 & 0,82 & 32 & 50,00 & 0,42 & 0,82 \\
\hline & 6 & 93,20 & 0,39 & 0,82 & 20 & 60,68 & 0,04 & 0,83 & 33 & 42,72 & 0,45 & 0,82 \\
\hline & 7 & 93,20 & 0,27 & 0,83 & 21 & 30,10 & 0,15 & 0,83 & 34 & 44,66 & 0,50 & 0,82 \\
\hline & 8 & 86,89 & 0,36 & 0,82 & 22 & 69,90 & 0,28 & 0,83 & 35 & 53,88 & 0,38 & 0,82 \\
\hline & 9 & 88,35 & 0,24 & 0,83 & 23 & 47,57 & 0,36 & 0,82 & 36 & 54,85 & 0,44 & 0,82 \\
\hline & 10 & 96,60 & 0,23 & 0,83 & 24 & 60,19 & 0,25 & 0,83 & 37 & 36,41 & 0,40 & 0,82 \\
\hline & 11 & 80,10 & 0,28 & 0,82 & 25 & 50,97 & 0,17 & 0,83 & 38 & 22,33 & 0,30 & 0,82 \\
\hline & 12 & 72,33 & 0,33 & 0,82 & 26 & 45,63 & 0,44 & 0,82 & 39 & 25,24 & 0,26 & 0,83 \\
\hline & 13 & 66,50 & 0,20 & 0,83 & 27 & 56,80 & 0,35 & 0,82 & 40 & 25,73 & 0,30 & 0,82 \\
\hline & 14 & 78,64 & 0,39 & 0,82 & & & & & & & & \\
\hline \multirow{5}{*}{ 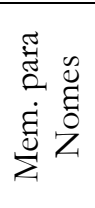 } & 1 & 99,03 & 0,12 & 0,62 & 6 & 97,09 & 0,21 & 0,61 & 11 & 52,91 & 0,55 & 0,53 \\
\hline & 2 & 100,00 & 0,00 & 0,62 & 7 & 61,65 & 0,35 & 0,59 & 12 & 16,02 & 0,29 & 0,60 \\
\hline & 3 & 99,03 & $-0,06$ & 0,63 & 8 & 61,65 & 0,40 & 0,57 & 13 & 3,88 & 0,21 & 0,61 \\
\hline & 4 & 98,54 & 0,14 & 0,62 & 9 & 74,27 & 0,30 & 0,60 & 14 & 4,37 & 0,19 & 0,61 \\
\hline & 5 & 97,09 & 0,11 & 0,62 & 10 & 13,11 & 0,35 & 0,59 & 15 & 0,97 & 0,20 & 0,62 \\
\hline
\end{tabular}

$N=206$

O teste Informação Geral apresentou um coeficiente de consistência interna (alpha total) igual a 0,95 , indicando que os itens do teste estão significativamente Psico-USF, v. 10, n. 2, p. 129-139, jul./ dez. 2005 correlacionados entre si, havendo coerência entre eles. De acordo com a Tabela 4 , em relação às porcentagens de acertos, dos 49 itens, 7 obtiveram porcentagens de 
acerto entre 80 e 100, 9 itens tiveram entre 60\% e $80 \%$ de acertos, 11 apresentaram porcentagens de acerto entre 40 e 60, 11 tiveram entre $20 \%$ e $40 \%$ de acertos e 11 itens possuíram porcentagens de acerto entre $0 \%$ e $20 \%$.

No que se refere aos 40 itens do teste Indução, a Tabela 5 sugere que 11 obtiveram porcentagens de acerto entre 80 e 100, 9 itens tiveram entre $60 \%$ e $80 \%$ de acertos, 15 apresentaram porcentagens de acerto entre 40 e 60, 5 itens tiveram entre $20 \%$ e $40 \%$ de acertos e nenhum possuiu porcentagens de acerto entre 0 e 20 . $\mathrm{O}$ coeficiente alpha total foi 0,83 , indicando que os itens do teste estão significativamente correlacionados entre si.

No Memória para Nomes, dentre os 15 itens, 6 obtiveram porcentagens de acerto entre 80 e 100 , 3 tiveram entre $60 \%$ e $80 \%$ de acertos, 1 item apresentou porcentagem de acerto entre 40 e 60, nenhum item possuiu porcentagem de acerto entre 20 e 40 e 5 apresentaram porcentagens entre 0 e 20 . O coeficiente alpha total em 0,70 indicou haver coerência entre os itens.

Tabela 5 - Desempenho em matemática e vocabulário geral: porcentagem de acertos, correlações item-total e coeficientes de consistência interna

\begin{tabular}{|c|c|c|c|c|c|c|c|c|c|c|c|c|}
\hline & Item & $\%$ & $\begin{array}{l}\text { Correl. } \\
\text { item-total }\end{array}$ & $\begin{array}{c}\text { Alpha se item } \\
\text { excluído }\end{array}$ & Item & $\%$ & $\begin{array}{c}\text { Correl. } \\
\text { item-total }\end{array}$ & $\begin{array}{l}\text { Alpha se item } \\
\text { excluído }\end{array}$ & Item & $\%$ & $\begin{array}{l}\text { Correl. } \\
\text { item-total }\end{array}$ & $\begin{array}{c}\text { Alpha se item } \\
\text { excluído }\end{array}$ \\
\hline \multirow{16}{*}{ 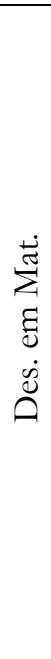 } & 1 & 97,07 & 0,22 & 0,95 & 17 & 62,44 & 0,69 & 0,95 & 33 & 32,68 & 0,59 & 0,95 \\
\hline & 2 & 94,63 & 0,36 & 0,95 & 18 & 79,02 & 0,50 & 0,95 & 34 & 44,88 & 0,62 & 0,95 \\
\hline & 3 & 94,63 & 0,27 & 0,95 & 19 & 69,27 & 0,63 & 0,95 & 35 & 31,22 & 0,57 & 0,95 \\
\hline & 4 & 90,73 & 0,39 & 0,95 & 20 & 49,76 & 0,64 & 0,95 & 36 & 20,49 & 0,56 & 0,95 \\
\hline & 5 & 93,17 & 0,38 & 0,95 & 21 & 43,41 & 0,74 & 0,95 & 37 & 30,24 & 0,57 & 0,95 \\
\hline & 6 & 92,20 & 0,10 & 0,95 & 22 & 75,12 & 0,62 & 0,95 & 38 & 20,98 & 0,53 & 0,95 \\
\hline & 7 & 81,46 & 0,43 & 0,95 & 23 & 32,20 & 0,50 & 0,95 & 39 & 25,37 & 0,52 & 0,95 \\
\hline & 8 & 84,88 & 0,33 & 0,95 & 24 & 49,76 & 0,60 & 0,95 & 40 & 13,66 & 0,46 & 0,95 \\
\hline & 9 & 52,20 & 0,48 & 0,95 & 25 & 44,88 & 0,62 & 0,95 & 41 & 04,39 & 0,24 & 0,95 \\
\hline & 10 & 65,37 & 0,56 & 0,95 & 26 & 31,71 & 0,59 & 0,95 & 42 & 10,24 & 0,42 & 0,95 \\
\hline & 11 & 78,54 & 0,61 & 0,95 & 27 & 64,39 & 0,56 & 0,95 & 43 & 51,71 & 0,64 & 0,95 \\
\hline & 12 & 74,15 & 0,65 & 0,95 & 28 & 62,93 & 0,62 & 0,95 & 44 & 60,98 & 0,57 & 0,95 \\
\hline & 13 & 81,95 & 0,39 & 0,95 & 29 & 57,07 & 0,57 & 0,95 & 45 & 48,29 & 0,62 & 0,95 \\
\hline & 14 & 64,39 & 0,69 & 0,95 & 30 & 30,24 & 0,58 & 0,95 & 46 & 45,85 & 0,60 & 0,95 \\
\hline & 15 & 79,51 & 0,56 & 0,95 & 31 & 45,85 & 0,68 & 0,95 & 47 & 20,00 & 0,53 & 0,95 \\
\hline & 16 & 76,10 & 0,53 & 0,95 & 32 & 47,32 & 0,69 & 0,95 & 48 & 3,90 & 0,26 & 0,95 \\
\hline \multirow{18}{*}{$\begin{array}{l}\overline{\widetilde{J}} \\
\dot{0} \\
\dot{0} \\
\dot{\tilde{U}} \\
0\end{array}$} & 1 & 78,64 & 0,57 & 0,93 & 19 & 55,34 & 0,61 & 0,93 & 36 & 25,48 & 0,46 & 0,93 \\
\hline & 2 & 75,24 & 0,49 & 0,93 & 20 & 37,13 & 0,52 & 0,93 & 37 & 25,48 & 0,43 & 0,93 \\
\hline & 3 & 68,93 & 0,52 & 0,93 & 21 & 57,76 & 0,58 & 0,93 & 38 & 50,48 & 0,59 & 0,93 \\
\hline & 4 & 71,11 & 0,46 & 0,93 & 22 & 64,80 & 0,11 & 0,94 & 39 & 16,99 & 0,59 & 0,93 \\
\hline & 5 & 86,41 & 0,31 & 0,94 & 23 & 36,65 & 0,63 & 0,93 & 40 & 42,96 & 0,64 & 0,93 \\
\hline & 6 & 91,26 & 0,23 & 0,94 & 24 & 34,71 & 0,62 & 0,93 & 41 & 20,63 & 0,64 & 0,93 \\
\hline & 7 & 85,43 & 0,30 & 0,94 & 25 & 38,35 & 0,64 & 0,93 & 42 & 7,76 & 0,43 & 0,93 \\
\hline & 8 & 57,76 & 0,55 & 0,93 & 26 & 58,98 & 0,51 & 0,93 & 43 & 10,19 & 0,49 & 0,93 \\
\hline & 9 & 81,07 & 0,18 & 0,94 & 27 & 72,08 & 0,32 & 0,94 & 44 & 12,38 & 0,39 & 0,93 \\
\hline & 10 & 86,16 & 0,25 & 0,94 & 28 & 31,07 & 0,66 & 0,93 & 45 & 62,13 & 0,48 & 0,93 \\
\hline & 11 & 73,54 & 0,43 & 0,93 & 29 & 23,54 & 0,45 & 0,93 & 46 & 4,85 & 0,36 & 0,93 \\
\hline & 12 & 75,73 & 0,50 & 0,93 & 30 & 71,60 & 0,60 & 0,93 & 47 & 6,55 & 0,44 & 0,93 \\
\hline & 13 & 47,08 & 0,32 & 0,93 & 31 & 49,03 & 0,36 & 0,94 & 48 & 59,22 & 0,45 & 0,93 \\
\hline & 14 & 61,16 & 0,60 & 0,93 & 32 & 38,10 & 0,63 & 0,93 & 49 & 58,01 & 0,41 & 0,93 \\
\hline & 15 & 69,41 & 0,48 & 0,93 & 33 & 67,72 & 0,25 & 0,94 & 50 & 68,69 & 0,32 & 0,94 \\
\hline & 16 & 70,63 & 0,42 & 0,93 & 34 & 14,80 & 0,57 & 0,93 & 51 & 81,79 & 0,39 & 0,93 \\
\hline & 17 & 63,59 & 0,42 & 0,93 & 35 & 52,91 & 0,42 & 0,93 & 52 & 85,68 & 0,39 & 0,93 \\
\hline & 18 & 41,50 & 0,58 & 0,93 & & & & & & & & \\
\hline
\end{tabular}

Um total de 48 itens caracterizou o teste Desempenho em Matemática, dos quais, de acordo com a Tabela 5, 9 itens obtiveram porcentagens de acerto entre 80 e 100, 12 itens tiveram entre $60 \%$ e $80 \%$ de acertos, 13 apresentaram porcentagens de acerto entre
40 e 60, 9 tiveram entre $20 \%$ e $40 \%$ de acertos e 5 itens possuíram porcentagens de acerto entre 0 e 20 . O coeficiente alpha total em 0,95 indica que os itens do teste estão significativamente correlacionados entre si, havendo coerência entre eles. 
Quanto ao Vocabulário Geral, dos 52 itens, 7 obtiveram porcentagens de acerto entre 80 e 100, 16 itens tiveram entre $60 \%$ e $80 \%$ de acertos, 12 apresentaram porcentagens de acerto entre 40 e 60,10 itens tiveram entre $20 \%$ e $40 \%$ de acertos e 7 possuíram porcentagens de acerto entre 0 e 20 . O coeficiente alpha total em 0,94 indica que os itens do teste estão significativamente correlacionados entre si.

Tabela 6 - Teste Vocabulário Ilustrado: porcentagem de acertos, correlações item-total e coeficientes de consistência interna

\begin{tabular}{|c|c|c|c|c|c|c|c|}
\hline Item & $\%$ & Correlação item-total & Alpha se item excluído & Item & $\%$ & Correlação item-total & Alpha se item excluído \\
\hline 1 & 100,00 & 0,00 & 0,94 & 48 & 42,23 & 0,52 & 0,93 \\
\hline 2 & 100,00 & 0,00 & 0,94 & 49 & 66,66 & 0,58 & 0,93 \\
\hline 3 & 100,00 & 0,00 & 0,94 & 50 & 88,83 & 0,32 & 0,93 \\
\hline 4 & 100,00 & 0,00 & 0,94 & 51 & 81,87 & 0,49 & 0,93 \\
\hline 5 & 100,00 & 0,00 & 0,94 & 52 & 58,73 & 0,67 & 0,93 \\
\hline 6 & 100,00 & 0,00 & 0,94 & 53 & 15,69 & 0,21 & 0,94 \\
\hline 7 & 100,00 & 0,00 & 0,94 & 54 & 49,03 & 0,42 & 0,93 \\
\hline 8 & 100,00 & 0,00 & 0,94 & 55 & 81,55 & 0,34 & 0,93 \\
\hline 9 & 99,03 & 0,10 & 0,94 & 56 & 69,09 & 0,30 & 0,94 \\
\hline 10 & 99,51 & 0,14 & 0,94 & 57 & 97,25 & 0,29 & 0,94 \\
\hline 11 & 98,54 & 0,21 & 0,94 & 58 & 90,61 & 0,39 & 0,93 \\
\hline 12 & 100,00 & 0,00 & 0,94 & 59 & 78,48 & 0,48 & 0,93 \\
\hline 13 & 100,00 & 0,00 & 0,94 & 60 & 74,59 & 0,25 & 0,94 \\
\hline 14 & 100,00 & 0,00 & 0,94 & 61 & 71,84 & 0,51 & 0,93 \\
\hline 15 & 99,03 & 0,07 & 0,94 & 62 & 83,98 & 0,23 & 0,94 \\
\hline 16 & 99,51 & $-0,01$ & 0,94 & 63 & 73,78 & 0,43 & 0,93 \\
\hline 17 & 100,00 & 0,00 & 0,94 & 64 & 59,22 & 0,65 & 0,93 \\
\hline 18 & 91,26 & 0,22 & 0,94 & 65 & 66,02 & 0,59 & 0,93 \\
\hline 19 & 99,51 & 0,16 & 0,94 & 66 & 66,34 & 0,55 & 0,93 \\
\hline 20 & 99,51 & 0,18 & 0,94 & 67 & 79,61 & 0,46 & 0,93 \\
\hline 21 & 92,07 & 0,21 & 0,94 & 68 & 44,17 & 0,45 & 0,93 \\
\hline 22 & 72,49 & 0,36 & 0,93 & 69 & 61,65 & 0,59 & 0,93 \\
\hline 23 & 89,96 & 0,53 & 0,93 & 70 & 23,78 & 0,45 & 0,93 \\
\hline 24 & 91,91 & 0,05 & 0,94 & 71 & 54,53 & 0,44 & 0,93 \\
\hline 25 & 53,23 & 0,44 & 0,93 & 72 & 69,41 & 0,50 & 0,93 \\
\hline 26 & 63,75 & 0,38 & 0,93 & 73 & 70,71 & 0,27 & 0,94 \\
\hline 27 & 97,08 & 0,10 & 0,94 & 74 & 17,47 & 0,45 & 0,93 \\
\hline 28 & 92,72 & 0,30 & 0,94 & 75 & 17,80 & 0,47 & 0,93 \\
\hline 29 & 93,69 & 0,16 & 0,94 & 76 & 48,05 & 0,55 & 0,93 \\
\hline 30 & 92,88 & 0,17 & 0,94 & 77 & 5,82 & 0,25 & 0,94 \\
\hline 31 & 98,54 & 0,19 & 0,94 & 78 & 39,48 & 0,49 & 0,93 \\
\hline 32 & 93,04 & 0,35 & 0,93 & 79 & 24,59 & 0,42 & 0,93 \\
\hline 33 & 95,14 & 0,35 & 0,93 & 80 & 9,71 & 0,39 & 0,93 \\
\hline 34 & 98,05 & 0,08 & 0,94 & 81 & 31,55 & 0,48 & 0,93 \\
\hline 35 & 90,45 & 0,43 & 0,93 & 82 & 47,89 & 0,57 & 0,93 \\
\hline 36 & 88,99 & 0,39 & 0,93 & 83 & 63,10 & 0,46 & 0,93 \\
\hline 37 & 68,93 & 0,54 & 0,93 & 84 & 90,45 & 0,24 & 0,94 \\
\hline 38 & 86,73 & 0,49 & 0,93 & 85 & 66,50 & 0,48 & 0,93 \\
\hline 39 & 77,18 & 0,22 & 0,94 & 86 & 72,33 & 0,52 & 0,93 \\
\hline 40 & 93,36 & 0,32 & 0,93 & 87 & 82,20 & 0,56 & 0,93 \\
\hline 41 & 76,86 & 0,40 & 0,93 & 88 & 96,44 & 0,26 & 0,94 \\
\hline 42 & 78,15 & 0,54 & 0,93 & 89 & 77,67 & 0,44 & 0,93 \\
\hline 43 & 79,12 & 0,40 & 0,93 & 90 & 44,17 & 0,51 & 0,93 \\
\hline 44 & 30,58 & 0,39 & 0,93 & 91 & 21,52 & 0,41 & 0,93 \\
\hline 45 & 95,14 & 0,32 & 0,94 & 92 & 46,92 & 0,41 & 0,93 \\
\hline 46 & 69,74 & 0,48 & 0,93 & 93 & 27,67 & 0,49 & 0,93 \\
\hline 47 & 38,35 & 0,52 & 0,93 & & & & \\
\hline
\end{tabular}

$N=206$ 
De acordo com a Tabela 6, dos itens do Vocabulário Ilustrado, 45 obtiveram porcentagens de acerto entre 80 e 100, 24 itens tiveram entre 60\% e 80\% de acertos, 12 apresentaram porcentagens de acerto entre 40 e 60, 7 tiveram entre $20 \%$ e $40 \%$ de acertos e 5 itens possuíram porcentagens de acerto entre 0 e 20 . O coeficiente alpha total em 0,94 indica que os itens do teste estão significativamente correlacionados entre si.

Não foi possível calcular os coeficientes de consistência interna dos testes Velocidade de Percepção e Rapidez de Resposta porque, para isso, haveria a necessidade do reteste ou da utilização da técnica das metades (Anastasi \& Urbina, 2000). As porcentagens de acerto também não foram calculadas nestes dois testes nem no "Memória Associativa", já que eles não foram caracterizados por itens de diversos níveis de dificuldade. No "Memória Associativa" o coeficiente de consistência foi 0,75 .

\section{Conclusões}

Uma constatação comum aos instrumentos que compuseram a BMI pode ser feita: a idade dos participantes teve uma influência altamente significativa sobre o desempenho em todos os testes elaborados. Tal fato parece indicar que o conjunto de testes mostrou-se sensível a mudanças desenvolvimentais, apontadas como um critério importante na verificação da validade de construto dos instrumentos (Anastasi \& Urbina, 2000).

Foi testada uma quantidade grande de questões para possibilitar a seleção de um conjunto de itens que atendesse aos critérios de adequada distribuição quanto à dificuldade e contribuição ao resultado total do teste. Uma das formas de verificar a adequação de um item é a análise da correlação que estabelece com o resultado total do teste (Pasquali, 2003). Porém, parece importante que a correlação do item com o resultado total seja estudada em conjunto com outros dados referentes ao item, como a porcentagem de acertos.

Por meio da análise das porcentagens de acerto e das correlações item-total foi possível elaborar a configuração final dos testes que compuseram a Bateria Multidimensional de Inteligência Infantil. Assim, o teste Informação Geral foi, em sua versão final, formado por 37 itens; o teste Indução por 28; o Memória para Nomes por 10; o teste Desempenho em Matemática por 39 itens. Finalmente, os testes Vocabulário Geral e Vocabulário Ilustrado foram compostos por 37 e 46 itens, respectivamente.

A análise dos itens apresentados à segunda amostra de participantes indicou que, de forma geral, os itens tiveram uma boa variância e se correlacionaram ao escore total. Apenas no teste Memória para Nomes houve a tendência a uma baixa correlação entre alguns itens e o escore total.

Quanto à precisão, foram encontrados coeficientes elevados nos testes Informação Geral, Indução, Desempenho em Matemática, Vocabulário Geral e Vocabulário Ilustrado. Nos testes Memória Associativa e Memória para Nomes os coeficientes tenderam a ser mais baixos, porém aceitáveis. Em relação aos testes Velocidade de Percepção e Rapidez de Resposta não foi possível calcular a consistência interna.

Finalmente, vale enfatizar a importância da realização de pesquisas com um número maior de participantes, incluindo outros estados do Brasil, e que também procurem compreender as relações entre o desempenho nas várias disciplinas escolares e as capacidades incluídas no modelo Cattell-Horn-Carroll. Da mesma forma seria relevante a investigação das capacidades gerais não enfatizadas no presente estudo: processamento visual, processamento auditivo, velocidade de decisão e leitura-escrita.

\section{Agradecimentos}

Os autores agradecem à Fundação de Amparo à Pesquisa do Estado de São Paulo (Fapesp).

\section{Referências}

Aiken, L. R. (2000). Psychological testing and assessment. Boston: Allyn and Bacon.

Almeida, L. S. (1988). Teorias da inteligência. Porto: Edições Jornal de Psicologia.

Anastasi, A. \& Urbina, S. (2000). Testagem psicológica. Porto Alegre, RS: Artes Médicas.

Campito, J. S. (1994). Verbal ability. Em R. J. Sternberg (Org.). Encyclopedia of human intelligence (pp. 1106-1115). New York: Macmillan.

Carroll, J. B. (1993). Human cognitive abilities: A survey of factor-analytic studies. New York: Cambridge University Press.

Cattell, R. B. (1987). Intelligence: Its structure, growth and action. Amsterdam: The Netherlands - Elsevier.

Cattell, R. B. (1998). Where is intelligence? Some answers from the triadic theory. Em J. J. McArdle \& R. W. Woodcock (Orgs.) Human cognitive abilities in theory and practice (pp. 29-38). New Jersey: Lawrence Erlbaum Associates.

Cronbach, L. J. (1996). Fundamentos da testagem psicológica. Porto Alegre, RS: Artes Médicas.

Flanagan, D. P. \& Ortiz, S. O. (2001). Essentials of crossbattery assessment. New York: John Wiley and Sons. 
Flanagan, D. P., Andrews, T. J. \& Genshaft, J. L. (1997). The functional utility of intelligence tests with special education populations. Em D. P. Flanagan, J. L. Genshaft \& P. L. Harrison (Orgs.). Contemporary intellectual assessment: Theories, tests, and issues. (pp. 457483). New York: The Guilford Press.

Friedman, L. (1995). The space factor in mathematics: Gender differences. Review of Educational Research, 65 (1), 22-50.

Gardner, H., Kornhaber, M. L. \& Wake, W. K. (1998). Inteligência: múltiplas perspectivas. Porto Alegre, RS: Artes Médicas.

Horn, J. L. (1991). Measurement of intellectual capabilities: A review of theory. Em K. S. McGrew, J. K. Werder \& R. W. Woodcock (Orgs.). Woodcock-Johnson technical manual (pp. 197-232). Chicago: Riverside.

Kline, P. (2000). Handbook of psycbological testing. London: Routledge.

Mayer, R. E. (2000). Intelligence and education. Em R. J. Sternberg (Org.). Handbook of intelligence (pp. 519-533). New York: Cambridge University Press.

McGrew, K. S. \& Flanagan, D. P. (1998). The Intelligence Test Desk Reference (ITDR) - Gf-Gc cross battery assessment. Boston: Allyn and Bacon.

Pasquali, L. (2003). Psicometria. São Paulo: Martins Fontes; PUCCamp.

Pinheiro, A. V. (1996). Contagem da freqüência de ocorrência de palavras expostas a crianças na faixa pré-escolar e séries iniciais do 10 grau. São Paulo: Associação Brasileira de Dislexia.

Rasanen, P. \& Ahonen, T. (1995). Arithmetic disabilities with and without reading difficulties: A comparison of arithmetic errors. Developmental Neuropsychology, 11(3), 275-295.

Schelini, P. W. (2002). Bateria Multidimensional de Inteligência Infantil: proposta de instrumento (Tese de Doutorado). Campinas, SP: Pontifícia Universidade Católica de Campinas.

Thorndike, R. M. (1997). The early history of intelligence testing. Em D. P. Flanagan, J. L. Genshaft \& P. L. Harrison (Orgs.). Contemporary intellectual assessment Theories, tests and issues (pp. 3-16). New York: The Guiford Press.

Recebido em maio de 2005

Reformulado em agosto de 2005

Aprovado em setembro de 2005

Sobre as autoras:

Patrícia Waltz Schelini é doutora em Psicologia pela Pontifícia Universidade Católica de Campinas e professora adjunta da Universidade Federal de São Carlos (UFSCar).

Solange Wechsler é doutora pela University of Georgia (Estados Unidos) e orientadora do Curso de PósGraduação em Psicologia da Pontifícia Universidade Católica de Campinas, onde coordena o Laboratório de Avaliação e Medidas Psicológicas - LAMP. 

Educacional

\author{
Universidade São Francisco - Programa de Pós-Graduação Stricto Sensu em Psicologia \\ http://docente.saofrancisco.edu.br/rprimi/ \\ e-mail labape@saofrancisco.edu.br
}

O LabAPE está ligado a duas das três linhas de pesquisa do Mestrado em Psicologia: Construção, validação e padronização de instrumentos de medida e Avaliação em Psicologia Educacional.

\title{
Os objetivos principais são:
}

1. Desenvolver e aprimorar instrumentos e técnicas de medida psicológica e educacional;

2. Investigar as aplicações práticas desses instrumentos em contextos educacionais, institucionais e organizacionais;

3. Desenvolver aplicações da Psicometria e a Teoria de Resposta ao Item;

4. Desenvolver recursos tecnológicos de informática que auxiliem a obtenção, organização, análise e interpretação de dados em psicologia e educação;

5. Organizar as informações coletadas através das pesquisas realizadas pelo grupo com o duplo propósito de (1) estabelecer parâmetros para os instrumentos pesquisados, (2) prover informações objetivas que auxiliem práticas preventivas e/ou interventoras dentro e fora da universidade.

\section{Financiamento: Fapesp e CNPq}

\section{Equipe}

Dr. Ricardo Primi (ricardo.primi@saofrancisco.edu.br)

Dra. Acácia Angeli dos Santos (acacia.santos@saofrancisco.edu.br)

Dra. Claudette Maria Medeiros Vendramini (claudette.vendramini@saofrancisco.edu.br)

Dra. Ana Paula Porto Noronha (ana.noronha@saofrancisco.edu.br) 